\title{
The effect of gesture on the perception of linearity in instrumental music
}

\author{
Samuel Gardner ${ }^{1 \dagger}$ \\ Vita Berezina-Blackburn ${ }^{1,2}$ \\ Daniel Shanahan ${ }^{1}$ \\ ${ }^{1}$ Ohio State University, Columbus, Ohio, USA \\ ${ }^{2}$ Advanced Computing Center for the Arts and Design, Ohio State University, Columbus, Ohio, USA \\ $\dagger$ Corresponding author: gardner.1162@,osu.edu \\ Published 16 December 2021; https://doi.org/10.18061/FDMC.2021.0033 \\ Author video presentation and/or other conference material: https://doi.org/10.17605/OSF.IO/U9WH5
}

\begin{abstract}
Given that music performances are made up of gestures, we might ask how the movements of an individual can alter how one perceives music. To address this question, this paper examines a hypothesis concerning the gestural priming of melodic events, and the role of this priming on the perceived continuation of the melody. When primed with a linear gesture, we hypothesize that participants will be more likely to select the continuation of a melodic idea, that is to say the melody keeps moving in the same direction. Conversely, when primed with circular gestures, participants will be more likely to select musical ideas that reverse and return to the starting pitch. Our results show that there was no significant effect of gesture, but there was a significant effect of musical scale when diatonic scales were used alongside the gesture. It appears that gestural priming is not a predictor of whether participants selected a musical gesture that continued or returned. These results suggest that familiarity with a musical context is perhaps more predictive of melodic expectation than gesture.
\end{abstract}

KEYWORDS: gesture, perception, motioncapture, melodic expectancy

\section{Introduction}

A musical performance comprises gestures, be they the performative gestures of playing an instrument, when a conductor leads an ensemble, when a dancer engages their entire body, or the responsive gestures one makes as they listen and perceive music. It would therefore seem that every aspect of making and engaging with music results in gestures. If gesture is important to how one makes and engages with music, can it inform one's understanding of music?

Previous research has discussed the role of crossmodal interactions between music and gesture. Schutz and Lipscomb (2007) found that, in regard to the marimba, "while longer gestures do not make longer notes, longer gestures make longer sounding notes through the integration of sensory information" (pg. 888). Previous research has also shown the importance of the visual modality when listening to music. Jane Davidson (1993) found that when a listener observes the musician performing when listening to music, that the visual perception more clearly specifies "manner" (defined as a way of doing a thing, in this case exaggerated, deadpan, and projected) than the other modalities. Similarly, Robert Gillespie (1997) finds that violin teachers, when only given the visual cue for vibrato, overcompensate on judging said vibrato, suggesting visual cues inadvertently influence their perception. The overall relationship between music and gesture is considered by Lawrence Zbikowski (2017) in his book Foundations of Musical Grammar, wherein he writes "[language and gesture are] built on an infrastructure of common conceptual ground and shared intentionality...music, puts greater emphasis on shared feelings and attitudes" (pp. 113-114). He claims that the shared "feelings and attitudes" have their basis in grounded bodily experience. It is this bodily experience that is considered in this paper.

Perhaps the most important claim of this paper is that gesture shapes thought. Most relevant to this particular study is the work of Barbra Tversky and her colleagues, particularly Tversky and Jamalian (2002) in which the authors found that gesture can alter how one perceives (non-musical) time as either linear or cyclical. In this study, participants were primed with gestures from the experimenter which were either depicting a line or a clock before being presented with a narrative. The participants were then asked to draw what they felt conveyed the narrative they were given, and in a second experiment they were asked what they felt came next. The results demonstrated that the gestural priming of a linear motion or a cyclical motion was highly predictive of the narrative selected. Similarly, Tversky's work with 
Seokmin Kang (2016) demonstrated that when gestures look like they thought that they represented, they can be even more powerful. If gestures are more effective when they resemble the linguistic goal, then this asks the question, are they also more effective when they resemble the musical goal? Susan Goldin-Meadow (2006) also writes about the importance of gestures in communication, arguing that when gesture is used on its own, it is able to function fully as communication, however, when used in tandem with language, gesture is then able to take imagistic forms, highlighting information not present in speech. This might be the case because, as David McNeill (1985) argues, gesture and speech are a part of the same "psychological structure" and that they share the same computational stage in thought (also see McNeill, 1992). Adam Kendon (1980) makes a similar argument that both gestures are "so intimately connected with the activity of speaking that we cannot say that one is dependent upon the other" (pg. 208).

Gesture is also shown to have an important role in explaining information that is difficult to conceptualize. In a study by Hostetter et al. (2007), they find that when participants are presented with a series of dots, or a series of dots and shapes, participants gesture more with the ambiguous series of dots in order to try to convey what they think the dots represent. Goldin-Meadow et al. (2009) found when learning a math lesson, nine and ten year olds who were forced to make the correct gesture with the problem learned more than those who were forced to make partially correct gestures, who in turn learned more than children who made no gestures.

Another aspect of the relationship between gesture and thought is the specific role of cognitive offloading. As Goldin-Meadow et al. (2001) are able to show, when participants are tasked with remembering a list of letters or words while forced to do another task, those participants who were able to gesture during their explanation of the task remembered more of the letters or words than those who were unable to gesture during the task. This suggests that gestures are able to allot more resources to memory than simply having to do the task. Further work continues to point to the power of gesture and cognitive load. For example, Ping and Goldin-Meadow (2010) show the role of gesture and cognitive load with objects that are not literally present, Wagner et al. (2004) shows that gesture is used significantly more in both visuospatial and verbal working memories, Gillespie et al. (2014) show that those with less working memory have higher rates of gesture, and Melinger and Kita (2007) highlight that gesture increases when the cognitive load needed for information processing is higher.

These ideas clearly place an importance on gesture and its role in communication, learning, and conceptualizing information, but gesture is also rooted in spatial thinking (for a detailed review see Alibali 2005; Hostteter and Alibali, 2008). One study by Ehrlich et al. (2006) shows that when children gestured about moving pieces when solving transformation puzzles, performed better than those who did not. Spatial orientation has recently been connected with musical motion and communication about music time. Cox (2016) for example argues that spatiality and music events/relationships are conceptually blended in the same mental domain (pg. 122-3). Godoy et al. (2016) take a similar approach and argue that it is shape similarity that is the common element in cross-modal research involving sound and space involving motion. Further, that, "for the implementation of shape cognition, we believe that body motion is necessary, and hence we locate the basis for amodal shape cognition in so-called motor theory. Motor theory is that which can encompass most (or most relevant) modalities by rendering whatever is perceived (features of sound, textures, motion, postures, scenes and so on) as actively traced shape images" (pg. 213).

\section{Method}

\section{Hypothesis}

We hypothesized that there would be a correspondence between the gestural priming condition (circular or linear gestures) and the perceived continuation of melodic tones. Put more formally:

H1a: When primed with linear gestures, participants will be more likely to select the continuation of melodic ideas (a continuously ascending scale, for example).

H1b: Conversely, when primed with circular gestures, participants will be more likely to select musical ideas that return (such as a change in direction, with a melody ascending and then descending).

\section{Participants}

113 participants (47 Male, 58 Female, 1 participant who identified as non-binary, and 7 participants who preferred not to answer; $\mathrm{M}=24.01$ years; $\mathrm{SD}=10.87$ ) completed an experiment online. Some were given course credit, whereas others volunteered. We asked participants a question "What title best describes you? 
(professional musician, semi-professional musician, serious amateur musician, amateur musician, musicloving nonmusician, or nonmusician)", taken from the Ollen Musical Sophistication Index (Ollen, 2006). Zhang and Schubert (2019) identified this as the most predictive single question of musical ability. 16 participants described themselves as amateur musicians, 30 as serious amateurs, 13 as professional musicians, 46 as semi-professional, and 8 as music-loving nonmusicians.

\section{Procedure and Design}

The participants were split into four groups, based on two factors, audio and gesture. Half of the participants were played the examples with audio from a piano, and the other half were given the examples as sine tones. Within each of those groups they were then presented with the same five audio examples accompanied by a motion-capture avatar motioning their hand with either a moving line across its body, or by circling around its body. A Picture of this avatar is seen in FIGURE 1. Participants were then asked "I play for you a piece of music. I'd like you to think about this music while watching this video. I will then play two more examples, and I would like for you to choose the one that is closest to your expectation." All groups were played the same five melodies and were asked to choose what they think came next. Two examples were the Western diatonic major scale, one was based on the whole-tone scale, one was based on the octatonic scale, and the final example was taken from the thirteen note Bohlen-Pierce scale.

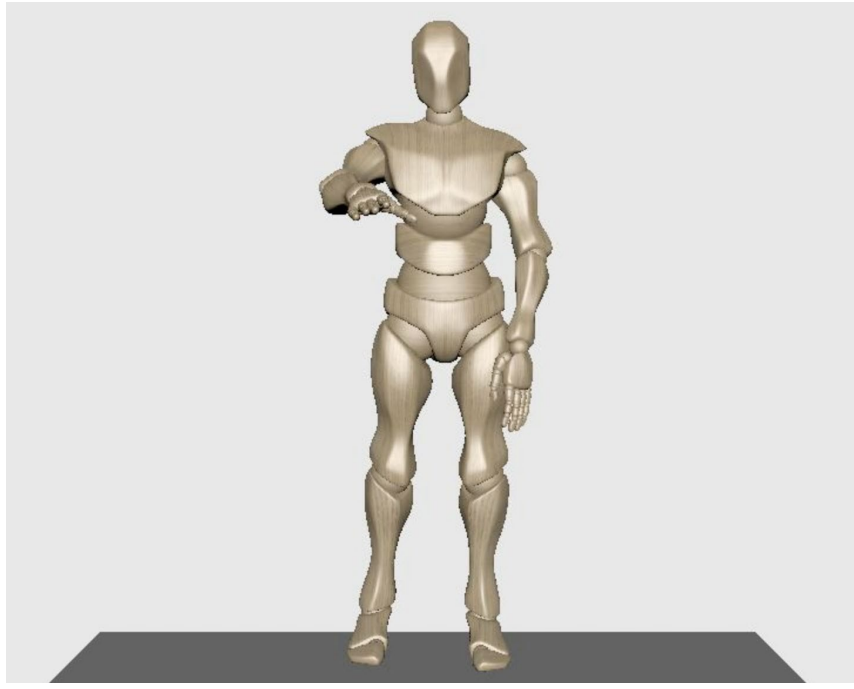

Figure 1: Motion-capture Avatar used in the experiment.

\section{Results}

Employing a multinomial logistic regression in which the participant response was predicted through both the gesture and the type of musical scale, we found no effect of gesture, but a significant effect of musical scale, specifically with the diatonic musical scale only $(\mathrm{p}=$ .006). It would appear that the gestural priming was not a predictor of whether participants selected a musical gesture that continued or returned.

Table 1: Results of a logistic regression predicting the correspondence of gesture and melodic idea.

\begin{tabular}{|l|l|l|l|l|}
\hline & $\begin{array}{l}\text { Estimat } \\
\text { e Std. }\end{array}$ & Error & $z$ Value & $\operatorname{Pr}(>|z|)$ \\
\hline Intercept & 0.4465 & 0.2347 & 1.902 & 0.05713 \\
\hline Linear & 0.2318 & 0.1826 & 1.269 & 0.20433 \\
\hline Diatonic & -0.7017 & 0.2573 & -2.728 & $\begin{array}{l}0.00638^{*} \\
*\end{array}$ \\
\hline Octatonic & -0.2811 & 0.2975 & -0.945 & 0.34476 \\
\hline $\begin{array}{l}\text { Whole } \\
\text { Tone }\end{array}$ & -.03396 & 0.2941 & -1.1155 & 0.24823 \\
\hline
\end{tabular}

\section{Discussion}

Judging from these results, it would seem that gesture does not play a significant role in the nature of melodic expectation, contrary to our hypotheses. There is a possibility that the prevalence of the major diatonic scale means that listeners are likely to infer a specific continuation, and that the priming plays no role in any scale whatsoever.

\section{Conclusion}

Future work will explore the interaction of musical events in such a way that listeners might be less accustomed to musical events, and will also explore the broader cross-cultural application of the effects of gesture on melodic expectation, as well as musical expectation more broadly.

\section{Acknowledgements}

This project could not have been completed without the support of Ohio State University's Center for Cognitive 
and Brain Sciences Summer Graduate Research Award. Support also comes from Ohio State University's Advanced Computing Center for the Arts and Design. Federico Camara-Halac assisted with the construction of the audio stimuli.

\section{References}

Alibali, M. W. (2005). Gesture in Spatial Cognition: Expressing, Communicating, and Thinking About Spatial Information. Spatial Cognition \& Computation, 5(4), 307-331. https://doi.org/10.1207/s15427633scc0504_2

Cox, Arnie. (2016). Music and Embodied Cognition. Indiana University Press. https://doi.org/10.2307/j.ctt200610s

Davidson, J. W. (1993). Visual Perception of Performance Manner in the Movements of Solo Musicians. Psychology of Music, 21(2), 103-113. https://doi.org/10.1177/030573569302100201

Ehrlich, S. B., Levine, S. C., \& Goldin-Meadow, S. (20061106). The importance of gesture in children's spatial reasoning. Developmental Psychology, 42(6), 1259. https://doi.org/10.1037/00121649.42.6.1259

Gillespie, M., James, A. N., Federmeier, K. D., \& Watson, D. G. (2014). Verbal working memory predicts co-speech gesture: Evidence from individual differences. Cognition, 132(2), 174-180. https://doi.org/10.1016/j.cognition.2014.03.012

Gillespie, R. (1997). Ratings of Violin and Viola Vibrato Performance in Audio-Only and Audiovisual Presentations. Journal of Research in Music Education, 45(2), 212-220. https://doi.org/10.2307/3345581

Godøy, R. I., Song, M., Nymoen, K., Haugen, M. R., \& Jensenius, A. R. (2016). Exploring Sound-Motion Similarity in Musical Experience. Journal of New Music Research, 45(3), 210-222. https://doi.org/10.1080/09298215.2016.1184689

Goldin-Meadow, S. (2006). Talking and Thinking With Our Hands. Current Directions in Psychological Science, 15(1), 34-39. https://doi.org/10.1111/j.0963-7214.2006.00402.x

Goldin-Meadow, S., Cook, S. W., \& Mitchell, Z. A. (2009). Gesturing Gives Children New Ideas About Math. Psychological Science, 20(3), 267-272. https://doi.org/10.1111/j.1467-9280.2009.02297.x

Goldin-Meadow, Susan, Nusbaum, Howard, Kelly, Spencer, \& Wagner, Susan. (2001). Explaining Math: Gesturing Lightens the Load. Psychological
Science, 12(6), 516-522. https://doi.org/10.1111/1467-9280.00395

Hostetter, A. B., \& Alibali, M. W. (2008). Visible embodiment: Gestures as simulated action. Psychonomic Bulletin \& Review, 15(3), 495-514. https://doi.org/10.3758/PBR.15.3.495

Hostetter, A. B., Alibali, M. W., \& Kita, S. (2007). I see it in my hands' eye: Representational gestures reflect conceptual demands. Language and Cognitive Processes, 22(3), 313-336. https://doi.org/10.1080/01690960600632812

Iverson, J. M., \& Thelen, E. (1999). Hand, mouth and brain. The dynamic emergence of speech and gesture. Journal of Consciousness Studies, 6(1112), 19-40.

Jamalian, A., \& Tversky, B. (n.d.). Gestures Alter Thinking About Time. 7.

Kang, S., \& Tversky, B. (2016). From hands to minds: Gestures promote understanding. Cognitive Research: Principles and Implications, 1(1), 4. https://doi.org/10.1186/s41235-016-0004-9

Kendon, A. (1980). Gesticulation and speech: Two aspects of the process of utterance. In The Relationship of Verbal and Nonverbal Communication (pp. 207-228). Walter de Gruyter. https://doi.org/10.1515/9783110813098.207

Kita, S. (2000). How Representational gestures help speaking. In Language and Gesture (pp. 162-185). Cambridge University Press. https://doi.org/10.1017/CBO9780511620850.011

McNeill, D. (n.d.). So You Think Gestures Are Nonverbal? 22.

McNeill, D. (1996). Hand and Mind: What Gestures Reveal about Thought (New edition). University of Chicago Press.

Melinger, A., \& Kita, S. (2007). Conceptualisation load triggers gesture production. Language and Cognitive Processes, 22(4), 473-500. https://doi.org/10.1080/01690960600696916

Ollen, J. E. (2006). A criterion-related validity test of selected indicators of musical sophistication using expert ratings

Ping, R., \& Goldin-Meadow, S. (2010). Gesturing Saves Cognitive Resources When Talking About Nonpresent Objects. Cognitive Science, 34(4), 602$619 . \quad$ https://doi.org/10.1111/j.15516709.2010.01102.x

Schutz, M., \& Lipscomb, S. (2007). Hearing Gestures, Seeing Music: Vision Influences Perceived Tone Duration. Perception, 36(6), 888-897. https://doi.org/10.1068/p5635 
Wagner, S. M., Nusbaum, H., \& Goldin-Meadow, S. (2004). Probing the mental representation of gesture: Is handwaving spatial? Journal of Memory and Language, 50(4), 395-407. https://doi.org/10.1016/j.jml.2004.01.002

Zbikowski, L. M. (2017). Foundations of Musical Grammar. Oxford University Press. https://doi.org/10.1093/oso/9780190653637.001.00 $\underline{01}$

Zhang, J. D., \& Schubert, E. (2019). A single item measure for identifying musician and nonmusician categories based on measures of musical sophistication. Music Perception: An Interdisciplinary Journal, 36(5), 457-467. https://doi.org/10.1525/mp.2019.36.5.457 